\title{
A GREY MODEL FOR SHORT-TERM PREDICTION OF THE IONOSPHERIC TEC
}

\author{
Hualin Shi ${ }^{1}$, Guiwen Lan ${ }^{1,2, *}$ \\ ${ }^{1}$ College of Geomatic Engineering and Geoinformatics, Guilin University of Technology, Guilin, China, China - (1434546262, \\ 23955461)@@qq.com \\ ${ }^{2}$ Guilin Research Centre for Engineering and Technology of Geomatics and Geoinformation Industry, Guangxi Beidouxing \\ Surveying and Mapping Science and Technology Co., Ltd, Guilin, Guangxi, China
}

KEY WORDS: Grey Model; Ionospheric TEC; Short-term Prediction; Optimization; Residual Error Correction; Improved Mode

\begin{abstract}
:
Accurate prediction of TEC can significantly improve the accuracy of navigation and positioning, therefore TEC observation and prediction has become a hot spot in ionospheric research. TEC has the characteristics of nonlinearity and non-stationarity, that cannot accurately describe this change by analytic expressions. Through the analysis of TEC content changes at the same time for several consecutive days in different seasons, it can be concluded that the TEC change at the same time in a short period is relatively stable, the overall monotonous change trend has a certain correlation. Since the grey model performs better in the prediction of a small amount of data and has a high accuracy in the prediction of time series with monotonous changes, it is used in the prediction of the same time and point-to-point short-term prediction of TEC. The accuracy of the grey model is verified by the Posterior variance ratio, the Little error probability test and the relation grade. The residual correction is made for the prediction results with low prediction accuracy, by further establishing the GM $(1,1)$ model of residual values, and the original prediction results being compensated and refined by the residual GM $(1,1)$ model. The experimental results show that the improved model is more accurate than the grey prediction model and can reflect the changing characteristics of ionospheric TEC.
\end{abstract}

\section{INTRODUCTION}

As one of the important characteristic parameters in the ionosphere, the Total Electron Content TEC (Total Electron Content) is directly related to the propagation error of satellite signals, and its Spatiotemporal changes will have an important impact on navigation, positioning, remote communication, etc.( MATTHEW,2008) Its observation and prediction have become a hot spot in ionospheric research(CAI,2006) (YUAN,2002)( MENG,2011) In the process of passing through the ionosphere, satellite signals will be affected by the instability of the ionosphere itself, resulting in signal delay, etc.( SCHMIDT,2007)Therefore, it is necessary to establish an appropriate model to analyse and predict the distribution of ionosphere electrons. (LI,2007) Currently, models for prediction of ionospheric TEC value are generally divided into two types: one type is empirical models based on statistical experience, such as Klobuchar, NeQuick, IRI model, etc.(CALAIS,1995)

Such models are widely used because of the advantages of simple calculation and easy to use. (LIU,2010)The other type of model is to use the existing observation data and use some mathematical modelling methods to extrapolate the ionospheric TEC, to obtain the prediction results. Modelling methods of such prediction models include time series model, artificial neural network model, (HABARULEMA,2007) autocorrelation analysis method, grey model, etc.( FAN,2010) The research shows that the above models can obtain good prediction results . ( RATNAM,2012)

Ionospheric TEC has obvious cyclical (24 h) and seasonal periodicity, day and night for days at the same time in different seasons of TEC content analysis found that, in a short period of time for a few days of TEC changes in the same time is relatively stable, the monotonous change trend overall, has certain correlation, can be monotonous change trend as the white part. However, the influence of the external environment can easily lead to random changes in ionospheric TEC, which can be regarded as the black part. Therefore, the change process of TEC at the same time in a few consecutive days in a short time can be regarded as a grey process, and GM $(1,1)$ model is adopted for prediction. (KAYACAN,2010)When the original ionospheric TEC sequence changes a lot, if the general GM $(1,1)$ model is directly used for prediction, the prediction results will often have a large deviation.( LU,2008) To solve this problem, this article through after effective differential ratio and small error probability test and correlation to test the accuracy of grey model, for accuracy is not high residual error of the grey model to predict the results of correction, establish the residual value of GM $(1,1)$ model(DENG,1982), through the residual error GM $(1,1)$ model prediction value compensation of the original forecast value, further elaboration prediction model, the ionosphere TEC forecast.

\section{GREY PREDICTION MODEL GM $(1,1)$}

\subsection{Establishment of Grey GM $(1,1)$ Model}

Grey forecasting is a forecasting method of grey system. Grey prediction identifies the degree of divergence of development trends among system factors, namely, carries out correlation analysis, and generates and processes the original data to find the law of system change, generates data sequences with strong regularity, and then establishes the corresponding differential equation model to predict the future development trend of things. (GUO,2012)

\footnotetext{
* Corresponding author
} 
The GM $(1,1)$ model was established with the ionospheric TEC sequence as the modelling sample. The modelling process was as follows:

1. the observed ionospheric TEC sequence is denoted as $x^{(0)}=\left\{x^{(0)}(1), x^{(0)}(2), \ldots, x^{(0)}(n)\right\}$. for1-AGO sequence is generated by a $x^{(0)}$ sum, denoted as $x^{(1)}=\left\{x^{(1)}(1), x^{(1)}(2), \ldots, x^{(1)}(n)\right\}$, where the sum formula is:

$$
x^{(1)}(k)=\sum_{i=1}^{k} x^{(0)}(i) \quad k=1,2, \ldots, n
$$

where

$$
\begin{aligned}
& k=1,2 \ldots, \mathrm{n} \\
& i=1,2 \ldots, \mathrm{n} \\
& x^{(0)}=\text { the observed ionospheric TEC sequence } \\
& x^{(1)}=1-\mathrm{AGO} \text { sequence is generated by a } x^{(0)} \text { sum }
\end{aligned}
$$

2. $z^{(1)}$ is the sequence generated by the immediate mean value of $x^{(1)}$, also known as the background value sequence, denoted as The calculation formula is as follows:

$$
\begin{aligned}
& z^{(1)}=\left\{z^{(1)}(2), z^{(1)}(3), \ldots, \quad z^{(1)}(n)\right\} \\
& z^{(1)}(k)=\frac{1}{2}\left(x^{(1)}(k)+x^{1}(k-1)\right), k=2, \cdots, n
\end{aligned}
$$

where

$$
\begin{aligned}
& z^{(1)}=\text { the sequence generated by the immediate mean } \\
& \text { value of } x^{(1)} \\
& k=2, \ldots, \mathrm{n} \\
& x^{(1)}=1 \text {-AGO sequence is generated by a } x^{(0)} \text { sum }
\end{aligned}
$$

3. The differential equation of the model $G M(1,1)$ is:

$$
\frac{d x^{(1)}}{d t}+a x^{(1)}=u
$$

where

$$
\begin{gathered}
x^{(1)}=1 \text {-AGO sequence is generated by a } x^{(0)} \text { sum } \\
\hat{a}=[a u]=\left(B^{T} B\right) B^{T} Y \\
B=\left[\begin{array}{cc}
-\frac{1}{2}\left(x^{(1)}(2)+x^{(1)}(1)\right) & 1 \\
-\frac{1}{2}\left(x^{(1)}(3)+x^{(1)}(2)\right) & 1 \\
\vdots \\
-\frac{1}{2}\left(x^{(1)}(n)+x^{(1)}(n-1)\right) & 1
\end{array}\right] \\
Y=\left[\begin{array}{c}
x^{(0)}(2) \\
x^{(0)}(3) \\
\vdots \\
x^{(0)}(n)
\end{array}\right]
\end{gathered}
$$

After we solve for $\hat{a}$, Solve the differential equation:

$$
\hat{x}_{1}^{(1)}(k+1)=\left[x^{(0)}(1)-\frac{u}{a}\right] e^{-a t}+\frac{u}{a}
$$

4. perform progressive reduction, and the reduction value of the model is:

$$
\hat{x}_{0}^{(1)}(k+1)=\hat{x}_{1}^{(1)}(k+1)-\hat{x}_{1}^{(1)}(k)
$$

5. calculate the prediction residual of the model and generate the residual sequence, denote as

$$
\begin{aligned}
& E=\left\{\varepsilon^{(1)}(1), \varepsilon^{(1)}(2), \cdots, \varepsilon^{(1)}(n)\right\} \\
& \varepsilon(k)=x^{(0)}(k)-\hat{x}^{(0)}(k) \quad k=1,2, \cdots, n
\end{aligned}
$$

\subsection{Accuracy Detection of Grey Model GM $(1,1)$}

The accuracy of grey model GM $(1,1)$ was tested using postverification square difference ratio $C$, small error probability test $P$ and correlation degree $W$. The calculation formulas of the three parameters are as follows:

1. difference ratio of the post-verification formula:

$$
C=S_{2} / S_{1}
$$

2. Small error probability:

$$
P=P(|\varepsilon(k)-\bar{\varepsilon}|)<0.6745 S_{1}
$$

3. correlation degree:

$$
W=\frac{1}{n} \sum_{i=0}^{n} \varepsilon_{i}(l)
$$

where

$$
\begin{aligned}
& S_{1}, S_{2}=\text { the variances of Sequence } \hat{x}^{(0)}(k) \text { and } \\
& \text { residual sequence } \varepsilon^{(0)}(k) \\
& \bar{\varepsilon}=\text { the mean of } \varepsilon^{(0)}(k) \\
& n=1,2 \ldots \mathrm{n} \\
& i=1,2 \ldots \mathrm{n}
\end{aligned}
$$

The posterior variance ratio $C$ more smaller, the larger the probability of small error $P$ is. Generally speaking, when $C$ is less than 0.35 and $P>0.95$, the higher the prediction accuracy of GM $(1,1)$ and the correlation degree $W>0.6$ indicate that the grey model GM $(1,1)$ has higher prediction accuracy.

\section{RESIDUAL GM $(1,1)$ MODEL}

As the growth of the time and the instability of the ionosphere TEC itself will lead to the grey model $\operatorname{GM}(1,1)$ prediction accuracy cannot be guaranteed, in order to improve the prediction precision of $\mathrm{GM}(1,1)$ model, in this paper, the prediction accuracy is not high residual error of the grey model to predict the results of correction, establish the residual value of GM $(1,1)$ model and residual error $\operatorname{GM}(1,1)$ model for short. The forecast value of residual GM $(1,1)$ model is used to 
compensate the original forecast value, so to further refine the forecast model and improve the prediction accuracy.

$$
\varepsilon^{(0)}=\left(\varepsilon^{(0)}(1), \varepsilon^{(0)}(2), \cdots \varepsilon^{(0)}(n)\right)
$$

where $\quad \varepsilon(k)=x^{(0)}(k)-\hat{x}^{(1)}(k)$ is the residual sequence of $x^{(1)}$

$n=$ the total number of samples

If there is $k_{0}$, it is satisfied

$$
\text { 1. } \forall k \geq k_{0}
$$

The symbol of $\varepsilon^{(0)}(k)$ is consistent

2.

$$
n-k_{0} \geq 4
$$

Then

$$
\left(\left|\varepsilon^{(0)}\left(k_{0}\right)\right|,\left|\varepsilon^{(0)}\left(k_{0}+1\right)\right|, \cdots\left|\varepsilon^{(0)}(n)\right|\right)
$$

is the mode label residual tail, still marked as

$$
\varepsilon^{(0)}=\left(\varepsilon^{(0)}\left(k_{0}\right), \varepsilon^{(0)}\left(k_{0}+1\right), \cdots \varepsilon^{(0)}(n)\right)
$$

Let

$$
\varepsilon^{(0)}=\left(\varepsilon^{(0)}\left(k_{0}\right), \varepsilon^{(0)}\left(k_{0}+1\right), \cdots \varepsilon^{(0)}(n)\right)
$$

be the mode label residual tail, The time response of the $\operatorname{GM}(1,1)$ model of the cumulative sequence

$$
\varepsilon^{(1)}=\left(\varepsilon^{(1)}\left(k_{0}\right), \varepsilon^{(1)}\left(k_{0}+1\right), \cdots \varepsilon^{(1)}(n)\right)
$$

is

$$
\hat{\varepsilon}^{(1)}(k+1)=\left(\varepsilon^{(0)}\left(k_{0}\right)-\frac{b_{\varepsilon}}{a_{\varepsilon}}\right) e^{\left[-a_{\varepsilon}\left(k-k_{0}\right)\right]}+\frac{b_{\varepsilon}}{a_{\varepsilon}}, k \geq k_{0}
$$

Then the simulation sequence of the residual tail is:

$$
\hat{\mathcal{\varepsilon}}^{(0)}=\left(\hat{\varepsilon}^{(0)}\left(k_{0}\right), \hat{\varepsilon}^{(0)}\left(k_{0}+1\right), \cdots \hat{\varepsilon}^{(0)}(n)\right)
$$

among them

$$
\hat{\varepsilon}^{(0)}(k+1)=\left(-a_{\varepsilon}\right)\left(\varepsilon^{(0)}\left(k_{0}\right)-\frac{b_{\varepsilon}}{a_{\varepsilon}}\right) e^{\left[-a_{\varepsilon}\left(k-k_{0}\right)\right]}, k \geq k_{0}
$$

Corrected by $\hat{\varepsilon}^{(0)}, \hat{X}^{(1)}$ is called modified time response

$$
\hat{x}^{(1)}(k+1)=\left\{\begin{array}{c}
\left(x^{(0)}(1)-\frac{b}{a}\right) e^{-a k}+\frac{b}{a}, k<k_{0} \\
\left(x^{(0)}(1)-\frac{b}{a}\right) e^{-a k}+\frac{b}{a} \pm a_{\varepsilon}\left(\varepsilon^{(0)}\left(k_{0}\right) \frac{b_{\varepsilon}}{a_{\varepsilon}}\right) e^{\left[-a_{\varepsilon}\left(k-k_{0}\right)\right]}, k \geq k_{0}
\end{array}\right.
$$

where

$$
\begin{aligned}
x^{(0)}(k) & =\text { The actual data } \\
\hat{x}^{(0)}(k) & =\text { Simulated data } \\
\varepsilon(k) & =\text { residual } \\
e & =\text { natural constant }
\end{aligned}
$$

The $\operatorname{GM}(1,1)$ model is modified for the residual, referred to as the residual $\operatorname{GM}(1,1)$. The sign of the residual correction value should be consistent with the sign of the residual tail $\varepsilon^{(0)}$.if

$$
\hat{x}^{(0)}(k)=\hat{x}^{(1)}(k)-\hat{x}^{(1)}(k-1)=\left(1-e^{a}\right)\left(x^{(0)}(1)-\frac{b}{a}\right) e^{-a(k-1)}
$$

Corresponding residual correction time responds.

$$
\hat{x}^{(0)}(k+1)=\left\{\begin{array}{c}
\left(1-e^{a}\right)\left(x^{(0)}(1)-\frac{b}{a}\right) e^{-a k}, \quad k<k_{0} \\
\left(1-e^{a}\right)\left(x^{(0)}(1)-\frac{b}{a}\right) e^{-a k} \pm a_{\varepsilon}\left(\varepsilon^{(0)}\left(k_{0}\right)-\frac{b_{\varepsilon}}{a_{\varepsilon}}\right) e^{\left[-a_{\varepsilon}\left(k-k_{0}\right)\right]}, \quad k \geq k_{0}
\end{array}\right.
$$

$$
\text { where } \quad \begin{aligned}
x^{(0)}(k) & =\text { The actual data } \\
\hat{x}^{(0)}(k) & =\text { Simulated data } \\
\varepsilon(k) & =\text { residual } \\
e & =\text { natural constant }
\end{aligned}
$$

Residual correction model called subtractive reduction.

\section{EXPERIMENTAL ANALYSIS}

\subsection{Data Selection}

According to the 11a cycle change of the number of sunspots, this paper selects the 2015 year of the year of the IGS centre102-115 (spring April), 193-206 (summer July), 282-295 (autumn October), 11-24 (Winter January) Ionospheric TEC grid point data at high latitude $\left(75^{\circ} \mathrm{N}, 115^{\circ} \mathrm{E}\right)$, mid-latitude $\left(40^{\circ} \mathrm{N}, 115^{\circ} \mathrm{E}\right)$, low latitude $\left(5^{\circ} \mathrm{N}, 115^{\circ} \mathrm{E}\right)$ as sample. Considering the inapplicability of the grey model $\operatorname{GM}(1,1)$ to a large amount of data, this paper uses the time-to-point prediction method and uses the ionospheric grid point observations released by the IIGS centre at intervals of $2 \mathrm{~h}$. The TEC data at the same time in the first 8 days is selected as the model sample, and the grey model $\operatorname{GM}(1,1)$ is used to predict the TEC value at the same time in the last 6 days, and the residual result is corrected for the prediction result of the grey model with low prediction accuracy. The $\operatorname{GM}(1,1)$ model of the residual value is established, and the original prediction value is compensated by the prediction value of the residual $\operatorname{GM}(1,1)$ model to further refine the prediction model. The forecast results are compared with the observations provided by IGS, and the accuracy of the model prediction is evaluated by defining the daily average relative accuracy $P$ and the root mean square error $R M S E$.

$$
\begin{aligned}
& p=\frac{1}{12} \sum_{i=1}^{12} 1-\frac{\left|I_{p}-I_{I G S}\right|}{I_{I G S}} \\
& R M S E=\sqrt{\frac{1}{12} \sum_{i=1}^{12}\left(I_{P}-I_{I G S}\right)^{2}}
\end{aligned}
$$

where

$$
I_{p}=\text { the forecast value, }
$$




$$
\begin{aligned}
& I_{I G S}=\text { the observation value issued by the IGS } \\
& \text { Center }
\end{aligned}
$$

$i=$ the number of 12 samples in a day.

\subsection{Forecast results and analysis}

The GM $(1,1)$ model with grey model and residual correction is used to forecast TEC data in different regions. The low latitude region is taken as an example. Figure 1 to Figure 4 shows the comparison between the prediction results of different models in the four latitudes in the low latitudes and the actual observation data. It can be seen that the prediction results of the four seasons in the low latitude region are overall, and the prediction accuracy in spring is better than that in the other three seasons. The accuracy is a bit worse.

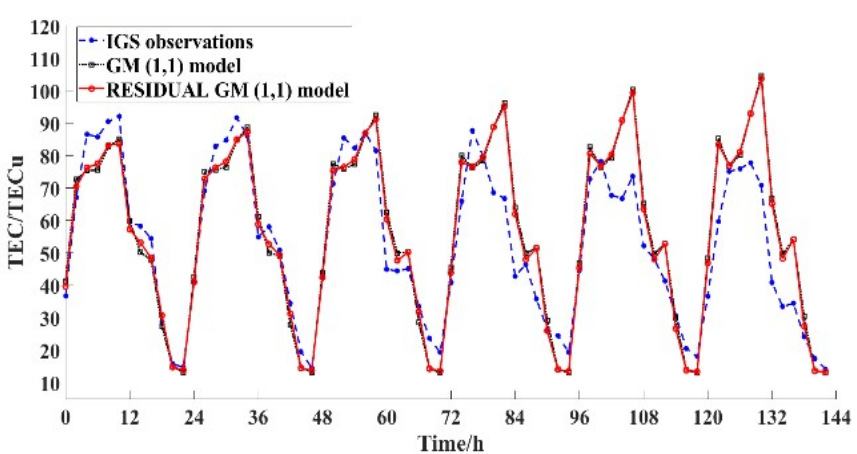

Figure 1. TEC forecast for 6 days in spring at low latitudes

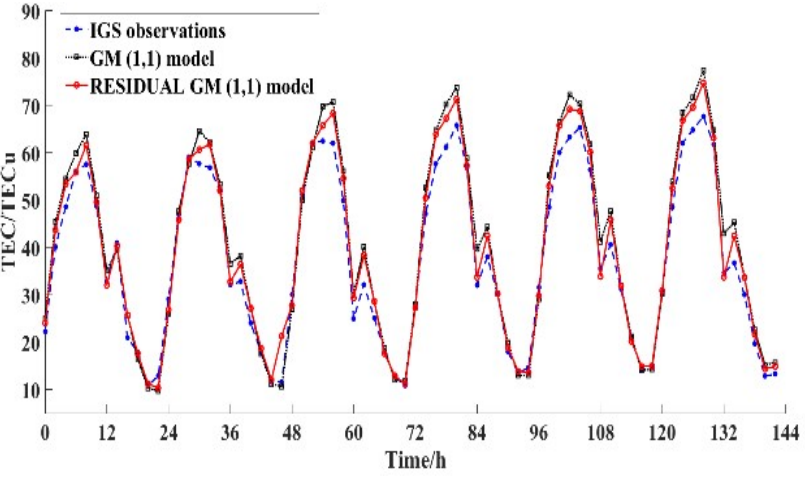

Figure 3. TEC forecast for 6 days in autumn at low latitudes

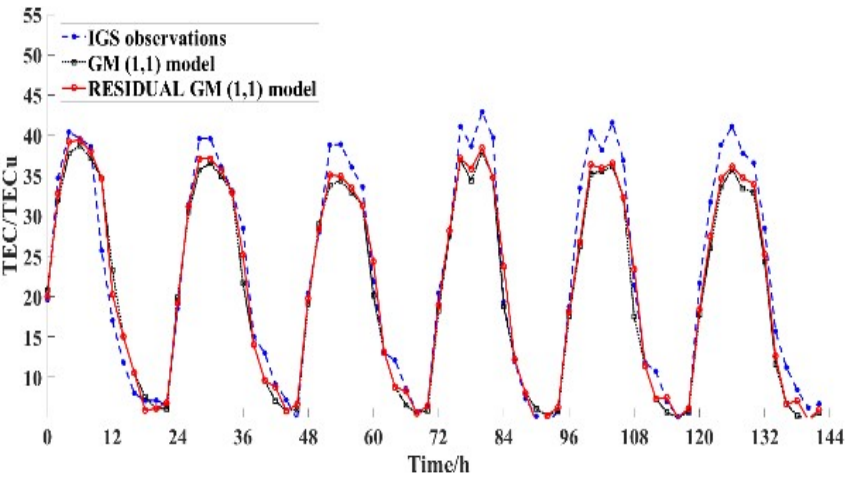

Figure 2. TEC forecast for 6 days in summer at low latitudes

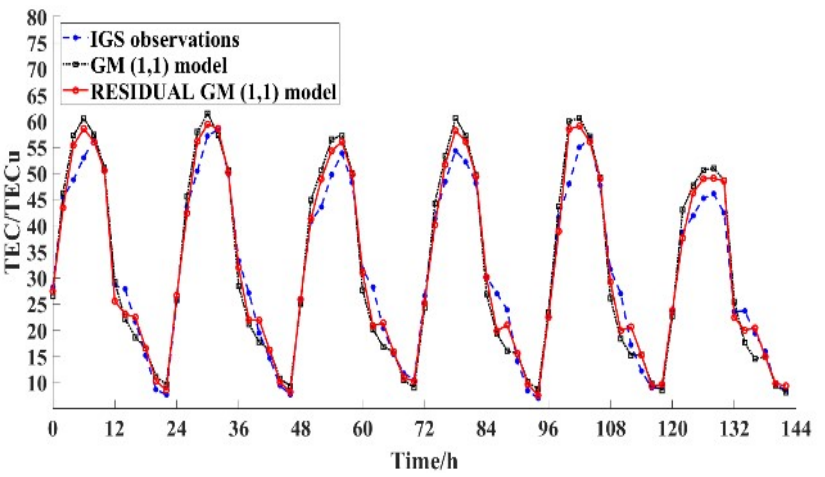

Figure 4. TEC forecast for 6 days in winter at low latitudes

\begin{tabular}{|c|cc|cc|cc|cc|}
\hline & \multicolumn{2}{|c|}{ Spring } & \multicolumn{2}{c|}{ Summer } & \multicolumn{2}{c|}{ Autumn } & \multicolumn{2}{c|}{ Winter } \\
Days & GM(1,1) & Residual GM & GM(1,1) & Residual GM & GM(1,1) & Residual GM & GM(1,1) & Residual GM \\
\hline 1 & 90.87 & 91.98 & 84.53 & 86.71 & 88.70 & 89.78 & 88.08 & 91.36 \\
2 & 89.75 & 92.54 & 86.89 & 89.41 & 89.51 & 85.66 & 89.79 & 93.12 \\
3 & 83.88 & 86.63 & 89.79 & 92.87 & 84.50 & 88.54 & 85.70 & 91.10 \\
4 & 74.36 & 76.87 & 86.42 & 84.85 & 86.91 & 91.26 & 83.62 & 89.80 \\
5 & 80.85 & 81.26 & 83.10 & 85.18 & 86.48 & 88.82 & 87.06 & 88.27 \\
6 & 68.70 & 71.20 & 71.02 & 76.10 & 78.44 & 83.29 & 82.11 & 85.59 \\
Mean & 81.40 & 83.41 & 83.63 & 85.85 & 85.76 & 87.89 & 86.06 & 89.87 \\
\hline
\end{tabular}

Table 1. Daily average relative accuracy of four models in low latitudes $\mathrm{P} \%$

\begin{tabular}{|l|cc|cc|cc|cc|}
\hline & \multicolumn{2}{|c|}{ Spring } & \multicolumn{2}{|c|}{ Summer } & \multicolumn{2}{c|}{ Autumn } & \multicolumn{2}{c|}{ Winter } \\
Days & $\mathrm{GM}(1,1)$ & Residual GM & $\mathrm{GM}(1,1)$ & Residual GM & $\mathrm{GM}(1,1)$ & Residual GM & $\mathrm{GM}(1,1)$ & Residual GM \\
\hline
\end{tabular}




\begin{tabular}{|c|ll|ll|ll|ll|}
\hline 1 & 85.63 & 90.22 & 87.57 & 90.37 & 91.12 & 92.46 & 86.60 & 92.05 \\
2 & 85.81 & 89.58 & 84.14 & 85.59 & 83.90 & 87.25 & 87.81 & 92.18 \\
3 & 92.53 & 96.24 & 83.54 & 84.97 & 80.77 & 83.15 & 78.52 & 86.25 \\
4 & 89.24 & 93.40 & 75.36 & 77.68 & 81.30 & 84.75 & 84.51 & 91.79 \\
5 & 91.24 & 94.65 & 90.04 & 91.47 & 76.84 & 80.07 & 81.21 & 87.78 \\
6 & 84.78 & 86.15 & 77.49 & 78.83 & 68.88 & 72.28 & 64.83 & 71.69 \\
Mean & 88.21 & 91.71 & 83.02 & 84.82 & 80.47 & 83.33 & 80.58 & 86.96 \\
\hline
\end{tabular}

Table 2. Daily average relative accuracy of four models in mid-latitudes $\mathrm{P} \%$

\begin{tabular}{|c|c|c|c|c|c|c|c|c|}
\hline \multirow[b]{2}{*}{ Days } & \multicolumn{2}{|r|}{ Spring } & \multicolumn{2}{|r|}{ Summer } & \multicolumn{2}{|c|}{ Autumn } & \multicolumn{2}{|r|}{ Winter } \\
\hline & $\operatorname{GM}(1,1)$ & Residual GM & $\operatorname{GM}(1,1)$ & Residual GM & $\operatorname{GM}(1,1)$ & Residual GM & $\operatorname{GM}(1,1)$ & Residual GM \\
\hline 1 & 83.02 & 90.80 & 89.28 & 92.50 & 71.62 & 80.43 & 76.85 & 85.91 \\
\hline 2 & 86.64 & 91.20 & 82.46 & 85.81 & 74.32 & 78.72 & 82.13 & 87.68 \\
\hline 3 & 87.44 & 90.77 & 79.09 & 82.08 & 74.66 & 78.46 & 68.40 & 75.66 \\
\hline 4 & 82.14 & 88.74 & 55.99 & 59.07 & 64.64 & 68.24 & 62.76 & 71.54 \\
\hline 5 & 79.12 & 87.46 & 58.67 & 61.27 & 66.59 & 73.91 & 75.77 & 77.64 \\
\hline 6 & 79.44 & 86.37 & 64.62 & 66.76 & 64.85 & 71.03 & 66.66 & 73.99 \\
\hline Mean & 82.97 & 89.22 & 71.69 & 74.58 & 69.45 & 75.13 & 72.10 & 78.74 \\
\hline
\end{tabular}

Table 3. Daily average relative accuracy of four models in high latitudes $\mathrm{P} \%$

Based on table 1, table 2 and table 3 prediction data to carry on the analysis of the daily average relative precision, after residual correction of grey model is relatively ordinary grey model in prediction accuracy on ascending to a certain extent, from the point of prediction area, low latitudes and mid-latitude every seasonal forecasting accuracy remains at a high level, and the forecast accuracy of change is relatively stable, the performance good prediction accuracy of mid-latitude spring 6 $\mathrm{d}$, daily average relative accuracy of $91.71 \%$. Except for $6 \mathrm{~d}$ in spring, the average daily relative accuracy of $6 \mathrm{~d}$ in high latitude area is $89.22 \%$, and the prediction accuracy of $6 \mathrm{~d}$ in other three seasons is all lower than $80 \%$, which is not ideal.

\begin{tabular}{|c|c|c|c|c|c|c|c|c|}
\hline Model & Area & $1 \mathrm{~d}$ & $2 \mathrm{~d}$ & $3 \mathrm{~d}$ & $4 \mathrm{~d}$ & $5 \mathrm{~d}$ & $6 \mathrm{~d}$ & Mean \\
\hline \multirow{3}{*}{$\mathrm{GM}(1,1)$} & Low latitude & 2.68 & 2.24 & 2.26 & 2.96 & 2.51 & 3.56 & 2.70 \\
& Middle latitude & 0.67 & 0.69 & 0.48 & 0.50 & 0.52 & 0.72 & 0.60 \\
& High latitudes & 1.75 & 1.54 & 1.41 & 1.86 & 2.54 & 2.67 & 1.9 \\
& Low latitude & 2.13 & 2.18 & 2.15 & 2.87 & 2.39 & 3.05 & 2.46 \\
& Middle latitude & 0.53 & 0.61 & 0.51 & 0.47 & 0.41 & 0.65 & 0.53 \\
& High latitudes & 1.51 & 1.48 & 1.32 & 1.66 & 2.35 & 2.31 & 1.77 \\
\hline \multirow{2}{*}{ Residual GM } & & & & & & & & \\
\end{tabular}

Table 4. RMSE statistics of mean-variance in spring forecast

\begin{tabular}{|l|l|l|l|l|l|l|l|l|}
\hline Model & Area & $1 \mathrm{~d}$ & $2 \mathrm{~d}$ & $3 \mathrm{~d}$ & $4 \mathrm{~d}$ & $5 \mathrm{~d}$ & $6 \mathrm{~d}$ & Mean \\
\hline
\end{tabular}


The International Archives of the Photogrammetry, Remote Sensing and Spatial Information Sciences, Volume XLII-3/W10, 2020 International Conference on Geomatics in the Big Data Era (ICGBD), 15-17 November 2019, Guilin, Guangxi, China

\begin{tabular}{|c|l|c|c|c|c|c|c|c|}
\hline \multirow{2}{*}{$\mathrm{GM}(1,1)$} & Low latitude & 2.12 & 2.14 & 2.23 & 2.67 & 2.45 & 2.75 & 2.39 \\
& Middle latitude & 1.37 & 1.58 & 1.47 & 2.28 & 0.87 & 2.16 & 1.62 \\
& High latitudes & 1.74 & 1.68 & 1.98 & 7.85 & 7.14 & 6.75 & 4.52 \\
\hline \multirow{2}{*}{ Residual GM } & Low latitude & 2.08 & 1.98 & 1.84 & 2.12 & 2.32 & 2.15 & 2.08 \\
& Middle latitude & 1.33 & 1.47 & 1.52 & 2.17 & 0.77 & 2.08 & 1.57 \\
& High latitudes & 1.61 & 1.41 & 1.83 & 7.02 & 6.94 & 6.26 & 4.18 \\
& & & & & & & &
\end{tabular}

Table. 5 RMSE statistics of mean-variance in summer forecast

\begin{tabular}{|c|c|c|c|c|c|c|c|c|}
\hline Model & Area & $1 \mathrm{~d}$ & $2 \mathrm{~d}$ & $3 \mathrm{~d}$ & $4 \mathrm{~d}$ & $5 \mathrm{~d}$ & $6 \mathrm{~d}$ & Mean \\
\hline \multirow{3}{*}{$\mathrm{GM}(1,1)$} & Low latitude & 2.46 & 2.42 & 2.38 & 2.48 & 2.37 & 2.56 & 2.46 \\
& Middle latitude & 1.57 & 1.68 & 1.71 & 1.69 & 2.01 & 3.12 & 1.96 \\
& High latitudes & 2.01 & 2.57 & 2.61 & 5.21 & 3.01 & 3.54 & 3.16 \\
\hline \multirow{3}{*}{ Residual GM } & Low latitude & 2.17 & 2.04 & 2.37 & 2.34 & 2.22 & 2.31 & 2.24 \\
& Middle latitude & 1.51 & 1.58 & 1.62 & 1.54 & 1.98 & 3.01 & 1.87 \\
& High latitudes & 2.04 & 2.35 & 2.57 & 5.08 & 2.87 & 3.24 & 3.02 \\
\hline
\end{tabular}

Table. 6 RMSE statistics of mean-variance in autumn forecast

\begin{tabular}{|c|c|c|c|c|c|c|c|c|}
\hline Model & Area & $1 d$ & $2 \mathrm{~d}$ & $3 \mathrm{~d}$ & $4 d$ & $5 \mathrm{~d}$ & $6 \mathrm{~d}$ & Mean \\
\hline \multirow{3}{*}{$\operatorname{GM}(1,1)$} & Low latitude & 2.66 & 2.67 & 2.88 & 3.01 & 2.18 & 2.78 & 2.70 \\
\hline & Middle latitude & 1.23 & 1.31 & 2.75 & 1.67 & 2.02 & 8.95 & 3.0 \\
\hline & High latitudes & 4.26 & 2.58 & 8.68 & 9.12 & 4.87 & 8.21 & 6.29 \\
\hline \multirow{3}{*}{ Residual GM } & Low latitude & 2.45 & 2.61 & 2.78 & 2.91 & 2.05 & 2.34 & 2.52 \\
\hline & Middle latitude & 1.19 & 1.21 & 2.65 & 1.57 & 1.93 & 7.96 & 2.75 \\
\hline & High latitudes & 1.26 & 1.15 & 4.35 & 6.55 & 4.26 & 4.10 & 3.61 \\
\hline
\end{tabular}

Table 7. RMSE statistics of mean variance in winter forecast

Table 4-7 statistics RMSE of the predicted data. Through analysis it is concluded that with the increase of forecast days, two models in different latitude root mean square error of predicted value has increased, in combination with table $1 \sim 3$, found that the average relative precision of low latitudes $P$ overall than mid-latitudes, predicted value of mean square error in numerical higher than mid-latitudes, analysis the reason is due to the low latitudes by solar radiation, which leads to the region of TEC value is larger, even if the region's RMSE value is bigger, also can get a better daily average relative accuracy.

\section{CONCLUSION}

In this paper, the grey prediction model GM $(1,1)$ is selected, the prediction method of the same time and point-to-point is applied to TEC short-term prediction, the residual correction is made for the prediction results of the grey model with low 
prediction accuracy, and the prediction results are compared with the observed values provided by IGS centre, and the following conclusions are drawn:

1. The improved model makes up for the missing accuracy of GM $(1,1)$ model in the prediction of the random part of TEC data due to the residual correction in the prediction results, which makes the prediction accuracy better than before.

2. From the perspective of prediction time, with the increase of forecast days, the model's prediction accuracy shows a downward trend.

3. From the perspective of the prediction area, the prediction accuracy of all seasons in the low-latitude area and mid-latitude area remains at a high level, and the prediction accuracy changes steadily. Except spring, the forecast precision of other three seasons in high latitude area is not ideal.

\section{REFERENCES}

Cai, C., Gao, J. X., Li, Z. H., 2006. Monitoring seasonal variations of ionospheric TEC using GPS measurements. Geomatics and Information Science of Wuhan University, 31(5), 451-453.

YUAN, Y. B., 2002. Study on Theories and Methods of Correcting Ionospheric Delay and Monitoring Ionosphere Based on GPS. Wuhan: Institute of Geodesy and Geophysics of the Chinese Academy of Sciences.

MENG, Y., AN, J. C., WANG, Z. M., 2011. Spatial Distribution of Antarctic Ionosphere TEC Based on GPS. Acta Geodaetica et Cartographica Sinica, 40(1), 37-40.

SCHMIDT, M., 2007. Wavelet modeling in Support of IRI. Advances in Space Research,(39), 932-940.

MATTHEW, P. F., EVANS, A. N., 2008. An evaluation of interpolation techniques for reconstructing ionospheric TEC map. IEEE Transactions on Geoscience and Remote Sensing. IEEE Transaction on Geoscience and Remote Sensing,46(7), 2153-2164.

CALAIS, E., MINSTER, J. B., 1995. GPS detection of ionospheric TEC Perturbations Following the January17,1994, Northridge Earthquake. Geophysical Research Letters,22(9), 1045-1048.

FAN, G. Q., WANG, W., XI, X. N., 2010. Modelling of Ionosphere VTEC Using Generalized Regression Neural Network. Acta Geodaetica et Cartographica Sinica,39(1), 1621.

RATNAM, V. D., DINESH, V. B., TEJASWI, B., et al, 2012. TEC Prediction Model Using Neural Networks over a Low Latitude GPS Station. International Journal of Soft Computing and Engineering ,2(2), 2231-2307.

HABARULEMA, J. B., MCKINNELL, L. A., CILLIERS, P. J., 2007. Prediction of Global Positioning System Total Electron Content Using Neural Networks over South Africa. Journal of Atmospheric and Solar-terrestrial Physics,69(15),1842-1850.
LIU, X. D., SONG, L. J., YANG, X. H., et al, 2010. Predicting Short-dated Ionospheric TEC Based on Wavelet Neural Network. Hydrographic Surveying and Charting ,30(5),49-51.

AKYILMAZ, O., ARSLAN, N., 2008. An Experiment of Predicting Total Electron Content (TEC) by Fuzzy Inference Systems. Earth, Planets, and Space ,60(9), 967-972.

LI, Z. G., CHENG, Z. Y., FENG, C. G., et al, 2007. A Study of Prediction Models for Ionosphere. Chinese Journal of Geophysice,50(2),327-337.

LI, X. H., GUO, D. Z.,2011. Prediction of Ionospheric Total Electron Content Based on Semiparametric Autoregressive Model. Science of Surveying and Mapping, (2),740-749.

KAYACAN, E., ULUTAS, B., KAYNAK, O., 2010. Grey System Theory-based Models in Time Series Prediction. Expert Systems with Applications,5(1), 784-1 789.

LU, X. F., YANG, Z. Q., JIA, X. L., et al, 2008. Parameter optimization method of grey system theory for the satellite clock error predicating. Geomatics and Information Science of Wuhan University,33(5),492-495.

DENG, J. L., 1982. The grey control system. Journal of Huazhong Institute of Technology,10(3),9-18.

GUO, Z. H., HAN, B. M., ZHAO, J. S., 2012. Satellites clock bias forecast based on grey system and BP neural network. Journal of Shandong University of Technology Natural Science Edition,26(4),39-42. 